\title{
SOFTWARE AGENT AND CLOUD COMPUTING: A BRIEF REVIEW
}

\author{
Firas D. Ahmed ${ }^{1 *}$, Mazlina Abdul Majid ${ }^{1}$, Mohd Sharifuddin ${ }^{1}$, Aws Nasser Jaber ${ }^{2}$ \\ ${ }^{1}$ Faculty of Computer Systems \& Software Engineering, Universiti Malaysia Pahang, \\ 26300 Gambang, Pahang, Malaysia \\ ${ }^{2}$ College of Information Technology, Universiti Tenaga Nasional, \\ Kajang, Malaysia \\ *Email: firas_firstone@yahoo.com \\ Phone: +60122988541
}

\begin{abstract}
The merging of interests between Cloud applications which necessary require an intelligent software agent with elastic, dynamic, with independent behavior ability and multi-agent systems that need consistent distributed infrastructures can be resulted with new effective applications and systems. Cloud computing services offered by using large-scale infrastructures with elastic services and high-performance capability since these resources could be adjusted to end user and application needs. Cloud systems and infrastructures are offered by a service-oriented interface that provides computing resources using $\mathrm{X}$-as-a-service model to introduce cloud services on the pay-per-use model. Agent-based system is significant for the improving the use of software agents for boosting cloud service composition, service discovery, negotiation mechanism and several domains. Integrating these two computing paradigms enables cloud-computing systems to become more elastic, autonomic, and intelligent service's capability. Meanwhile, scalable systems with high-performance on the cloud are capable of providing MASs with a consistent and large-scale computing infrastructure on which to execute large-scale systems with flexibility. The significant of this paper is introducing and discussing potential benefits of integration multi-agent technology with Cloud computing systems. This reviewing will lead having in attention the aim of developing high-performance and sophisticated applications with intelligent using software agents and Cloud paradigm.
\end{abstract}

Keywords: Software Agent; Cloud Computing; Multi Agent Systems; Cloud Systems.

\section{INTRODUCTION}

Cloud computing offer scalable and elastic services with high performance to a huge number of customers such as providers and end users (Armbrust et al., 2010). Cloud computing is considered as a big step into the distribution technologies' arena by providing innovative Internet services, which consider as complementary to the distributed computing functions which provided by Grid computing, P2P networks, and Web 2.0, which enrich of development cloud applications and interfaces (Armbrust et al., 2010; Carlin \& Curran, 2012). Actually, Cloud infrastructure provides large-scale resources and services with high-performance that are automatically adapted to ending users and application necessities. Nowadays, Cloud systems are mainly nominated for hold increasingly demanding computing resources with providing a huge data-storage using a pay-per-use model. With these benefits are combined of possibly solving 
management difficulties and reducing cost's usage and increasing incomes (Carlin \& Curran, 2012).

Multi-agent systems that we will call it as (MASs) are considered as one of the distributed computing paradigms built based on interacting of agents, which behave intelligently to solve complex problems (Jagannathan et al., 2002). Multi agent systems can solve sophisticated problems using a distributed approach where a number of agents can reach the target by intelligent collaborating between each other. One of the significant features of software agents is the intelligently solving problems. This intelligent behavior can be embodied into software agents based on some AI approaches. Nevertheless, there is a need of several agent inter-cooperation's to run on distributed hosts such as servers or normal PCs to achieve goals with high-performance execution which we need for solving compound problems with sustain execution time low as could be possible (Nwana, Lee, \& Jennings, 1996).

Even if there are differences between Cloud computing and MASs, but definitely, both are classified as distributed technologies. Therefore, many similar problems can be shared between these two technologies, and many goals can be gained by the matching use of Cloud computing and MAS technologies (Sim, 2012; Talia, 2011). The stakeholders in the Cloud computing area are mainly concentrated on the efficiently use of the computing infrastructure, energy efficiency, service delivery, and big data-storage facilitation (Sim, 2012). So that, we can say the most focus of research activities in Cloud computing is on the efficient use of the infrastructure to reduce costs (Sim, 2012).

In contrast, research events within the area of agents are mostly concentrated on the intelligent parts of agents and their use to develop complex simulations and applications. Therefore, the main problems are concerned with issues such as distributed computational intelligence, compound simulation, software-intensive applications, and adaptive systems (Aversa, Di Martino, Rak, \& Venticinque, 2010; Talia, 2011). Although, Cloud computing and multi agent systems are sharing common issues and research topics. In both technologies, several correspondences need to be explored. Firstly, Cloud computing can provide high performance, elastic, and large-scale computing infrastructure for the MASs execution and implementation during development and simulation of complex systems (Aversa, Di Martino, Rak, \& Venticinque, 2010; Sim, 2012; Talia, 2011). Secondly, MASs can be considered as a basic component for offering intelligence services in Cloud computing systems, which make cloud services more adaptive, flexible, and autonomously scheduling and allocation resources and provisioning it in running large-scale applications (Nwana, Lee, \& Jennings, 1996). For these causes and others, this paper introduces research ideas in these two areas and points out potential interactions that deserve to investigated and analyzed. This covering is led having in mind the goal of implementing highperformance complex systems and intelligent applications by using both Cloud computing systems and software agents. In next sections, we will make focus in cases of integration between software agents and cloud computing paradigms. Last section concludes the paper.

\section{CLOUD USING SOFTWARE AGENTS}

Cloud computing consider as a new distributed advancement which has been investigated and applied in the last five years (Armbrust el al., 2010). Cloud computing appears as a new paradigm because of big-business companies that were observing to a 
large-scale computing infrastructure for delivery service-oriented products with low costs (Sim, 2009). Many of the current developments on Cloud computing infrastructures and services were dedicated to the discovery of Cloud computing infrastructures and key technologies such as virtualization and service oriented interfaces. Meanwhile, slight research activities have been focusing to present innovative methods for both of beneficial users; customers and providers to discover, composite, manage and use cloud resources effectively (Armbrust, et al, 2010; Sim, 2009). MASs considered as a suitable solution for authorizing user access and control, managing the cloud resources and service composition automatically, and service discovery, marketing, and prices automotive determining of Cloud resources (Aversa, Di Martino, Rak, \& Venticinque, 2010; Talia, 2011) A new paradigm, denoted as agentbased cloud computing comes out to provide agent-based solutions. Agent-based cloud computing was founded to develop software agents for enhancement cloud resources management, service discovery, service composition and SLA negotiation (Sim, 2012). Agents with Intelligent behavior can make cloud systems smarter through the interaction between agents with users, and more efficient in allocating computing resources and big data storage. In scalable server's farms, agents can execute many tasks such as searching, filtering, querying and updating data, which are stored in these super servers (Sim, 2009). Let us imagine the expected benefits when cloud agents employed on operating systems and our behalf, to provide monitoring services, access authentication services, and energy-saving power of Cloud infrastructures (Aversa, Di Martino, Rak, \& Venticinque, 2010). Such research activities and much more efforts should be implemented to develop agent-based solutions for Cloud computing services. Such efforts to the main three X-as-a-Service delivery and deployment models should be done. In IaaS, software agents are beneficial for supporting the resources provisioning to user applications intelligently (Aversa, Tasquier, \& Venticinque, 2012) In PaaS, Software agent can participate in deployment and runtime executing of programming platforms that developers use for application developing efficiently Aversa, Di Martino, Rak, \& Venticinque, 2010). In SaaS, MASs could be a suitable way to optimize the applications use, which provided on demand as services, clouds could be an effective choice for the underlying hardware infrastructure and software runtime management, and its effective utilization with keeping the QoS as declared to end users (Jagannathan et al., 2002). In Clouds, there also need to invent approaches that adjust to the autonomic behaviors of Cloud computing services (Venticinque, Tasquier, \& Martino, 2012). This autonomy characteristic, which available in software agent technology help service's customers to reach this goal. MASs ability to handle with changing volatility, configurations, heterogeneity can offer an effective way for solve these requirements. Finally yet importantly, trustiness and security issues are two serious challenges in Cloud systems because confidential data and private software are hosted, accessed, and run on servers, which are not controlled by the end users directly (Sim, 2009). In such situation, agent-based models with trustiness and security algorithms in Cloud infrastructures could be significant to overcome such challenges (Lopez-Rodriguez \& Hernandez-Tejera, 2011). In rapid, if agent-based technologies such as intelligent behavior, autonomic, smart and learning, and negotiation techniques are involved in the Cloud infrastructures and services, we will have:

- $\quad$ Cloud services with Intelligent and flexible capability,

- Cloud services with Autonomous and pro-active characteristics,

- $\quad$ Cloud services Managed Autonomously. 


\section{SOFTWARE AGENTS USING CLOUDS}

In most cases, the scalable agent-based systems or simulations, which are based on Multi-agents Paradigm, require large data-storage computing and high-performance capability. In this way, Cloud based infrastructure can consider as an optimal solution where to implement agent-based systems and simulations because of its offering scalable and easy controllable and manageable computing resources, which can be configured easily for running agent-based software and dynamically (Lopez-Rodriguez \& Hernandez-Tejera, 2011). MASs can use cloud computing infrastructures to access the huge amount of resources such as processors and data. MASs and its applications can be hosted on a Clouds entirely or just the most demanding part of it can be run and host in the Clouds (Talia, 2011). Meanwhile, other parts can be hosted and run on a local infrastructure. Consequently, Multi agents can be more effective, lighter with smarter behavior to run and implement it tasks easily. In addition, MASs can increase their accurateness and intelligence by implementing algorithms, which are more sophisticated to run it by limited and traditional platforms without high-performance infrastructure where to be implemented in (Buyya, Vecchiola, \& Selvi, 2013).

In fact, the amount of computing resources such as processing power and data storage of a Cloud could enable MASs to become greater than other distributed computing paradigms, with more flexible with high performance. Cloud based systems can embed agents, with its dynamic and large-scale capability, and these distributed computing technologies leading to new solution to agent computing applications and enriching agent's knowledge-base more than conventional computing technologies, which are offered in these days (Buyya, Vecchiola, \& Selvi, 2013; Kephart, \& Chess, 2003). Virtualization technologies which is the underlying base for Cloud computing, which can be considered as an effective resource composition way for parallel virtual machines were to execute large-scale and concurrent agents which needing high performance for achieving tasks in a timely manner (Kephart, \& Chess, 2003). In cloudbased systems, Agents can be adapted to available VMs with using features of agents such as, pro-activity, learning, and autonomy. One of the most significant properties of Clouds is elasticity; which means the ability of expanding and shrinking resources based on users or applications demand (Buyya, Vecchiola, \& Selvi, 2013). This characteristic is significant for MASs in execution of scalable simulations and sophisticated applications to be able for adapting the available resources. Finally, software agent can find the ideal platform where to run and access resources such as servers, and large data, in Cloud computing infrastructures (Aversa, Tasquier, \& Venticinque, 2012). These benefits and the significant role of software agent using cloud must be subjugated for developing an efficient MASs. Therefore, software agents using cloud led to advances on the techniques of developing a new generation of largescale MASs (Buyya, Vecchiola, \& Selvi, 2013).

\section{CONCLUSION}

We conclude such integration between Cloud computing and MASs could be considered as a suitable solution for both paradigm's issues. We introduced and discussed through this paper, which research areas should involve investigating benefits of using the software agent, and cloud that led to intelligent cloud services and highperformance capability. This merging of interests between Cloud systems that require 
intelligent problem solving capability with dynamic, elastic, and independent behavior and multi-agent systems that need consistent distributed infrastructures can lead to produce new effective systems and applications. In cooperation with the agent and cloud research experts, this opportunity should be explored together with implementing joint research activities that needed to gain this goal. The research topics such as service discovery, service composition, resources with dynamic scheduling, and allocation for cloud computing resources, VMs and service migration, energy aware resource and service SLA negotiation must be included within the research plan for agent-based cloud computing. The expected benefits from the integration of agents and clouds could be many and significant. In conclude, we could have intelligent cloud services using MASs in the future, and MASs can get benefits from cloud infrastructure and computing resources to facilitate and support the implementation and simulation for large-scale agent-based applications.

\section{ACKNOWLEDGEMENTS}

We would like to thank Domenico Talia, for his advices on this research opportunity and reviewing it and special thanks to IPS/UMP for their supporting to postgraduate students.

\section{REFERENCES}

Armbrust, M., Armando, F., G, Anthony, D. J., Randy, K., Andy K., Gunho, L. (2010). A view of cloud computing. Communications of the ACM 2010, 50-58.

Aversa, R., Beniamino, D. M., Massimiliano, R., \& Salvatore, V. (2010). Cloud agency: A mobile agent based cloud system. In Complex, Intelligent and Software Intensive Systems (CISIS), IEEE International Conference. 132-137.

Aversa, R., Tasquier, L., \& Venticinque, S. (2012). Management of cloud infrastructures through agents. In Emerging Intelligent Data and Web Technologies (EIDWT), IEEE Third International Conference, 46-53.

Buyya, R., Christian, V., \& Thamarai, S. S. (2012). Mastering cloud computing. Tata McGraw-Hill Education.

Jagannathan, S., Richard, A. K., James, F. P., Satoru, F., Kazuya, K., \& Toru, Y. (2002). Distributed agent software system and method having enhanced process mobility and communication in a computer network. U.S. Patent 6.

Kephart, J. O., \& David, M. C. (2013).The vision of autonomic computing. Computer $36(1), 41-50$.

Lopez-Rodriguez, I., \& Mario, H. (2011). Software agents as cloud computing services. In Advances on Practical Applications of Agents and Multi agent Systems, Springer Berlin Heidelberg.

Nwana, H. S., Lyndon, C. L., \& Nicholas, R. J. (1996). Coordination in software agent systems. British Telecom Technical Journal 14 (4), 79-88.

Sim, K. M. (2009). Agent-based cloud commerce. In Industrial Engineering and Engineering Management, IEEE International Conference, 717-721.

Sim, K. M. (2012). Agent-based cloud computing. Services Computing, IEEE Transactions, 564-577.

Talia, D. (2012). Clouds meet agents: Toward intelligent cloud services. IEEE Internet Computing 16(2), 78-81. 
Talia, D. (2012). Cloud Computing and Software Agents: Towards Cloud Intelligent Services. In WO, 11(1), 2-6.

Venticinque, S., Luca, T., \& Beniamino, D. M. (2012). Agents based cloud-computing interface for resource provisioning and management. In Complex, Intelligent and Software Intensive Systems (CISIS), Sixth International Conference on 2012, 249-256.

Weinman, J. (2011).The Future of Cloud Computing. In Technologies Beyond 2020 (TTM), IEEE Technology Time Machine Symposium 2011, 1-2. 